Supporting Information for

\title{
Hierarchically Ordered Structures of Rod-Rod Block Copolymers Containing Two Mesogen-Jacketed Liquid Crystalline Polymers
}

Zhehao Tang , Ping-Ping Houł, Wei Zhang, Xiaolin Lu, Zhihao Shen*, and Xing-He Fan*

Beijing National Laboratory for Molecular Sciences, Key Laboratory of Polymer Chemistry and Physics of Ministry of Education, Center for Soft Matter Science and Engineering, College of Chemistry and Molecular Engineering, Peking University, Beijing, 100871, China

${ }^{\dagger}$ These authors contributed equally.

* To whom the correspondence should be addressed. E-mail: fanxh@pku.edu.cn (X.-H.F.); zshen@pku.edu.cn (Z.S.). 


\section{Results}

Table S1. Thermal analysis data of SmPn

\begin{tabular}{ccccc}
\hline \multirow{2}{*}{ polymer } & $T_{\mathrm{d}}\left({ }^{\circ} \mathrm{C}\right)^{a}$ & \multicolumn{3}{c}{$T_{\text {transition }}\left({ }^{\circ} \mathrm{C}\right)$} \\
\cline { 2 - 5 } & & peak 1 & peak 2 & peak 3 \\
\hline S10P30 & 378 & $-d$ & $135^{b}(0.301)^{c}$ & $175^{b}(2.84)^{c}$ \\
S20P30 & 373 & $64^{b}(0.430)^{c}$ & $-d$ & $169^{b}(1.70)^{c}$ \\
S30P30 & 370 & $60^{b}(0.509)^{c}$ & $139^{b}(0.158)^{c}$ & $185^{b}(2.34)^{c}$ \\
S30P120 & 365 & $67^{b}(0.266)^{c}$ & $145^{b}(2.90)^{c}$ & $201^{b}(7.08)^{c}$ \\
S80P120 & 362 & $64^{b}(0.974)^{c}$ & $149^{b}(2.15)^{c}$ & $195^{b}(3.48)^{c}$ \\
\hline
\end{tabular}

a $5 \%$ weight loss temperature determined by using TGA during heating at a rate of $10{ }^{\circ} \mathrm{C} / \mathrm{min}$ under nitrogen.

${ }^{b}$ Determined by DSC during the second heating process at $20^{\circ} \mathrm{C} / \mathrm{min}$.

$c$ Enthalpy changes $(\mathrm{kJ} / \mathrm{mol})$ of transitions obtained from DSC experiments during the second-heating process at $20^{\circ} \mathrm{C} / \mathrm{min}$.

${ }^{d}$ Not observed.
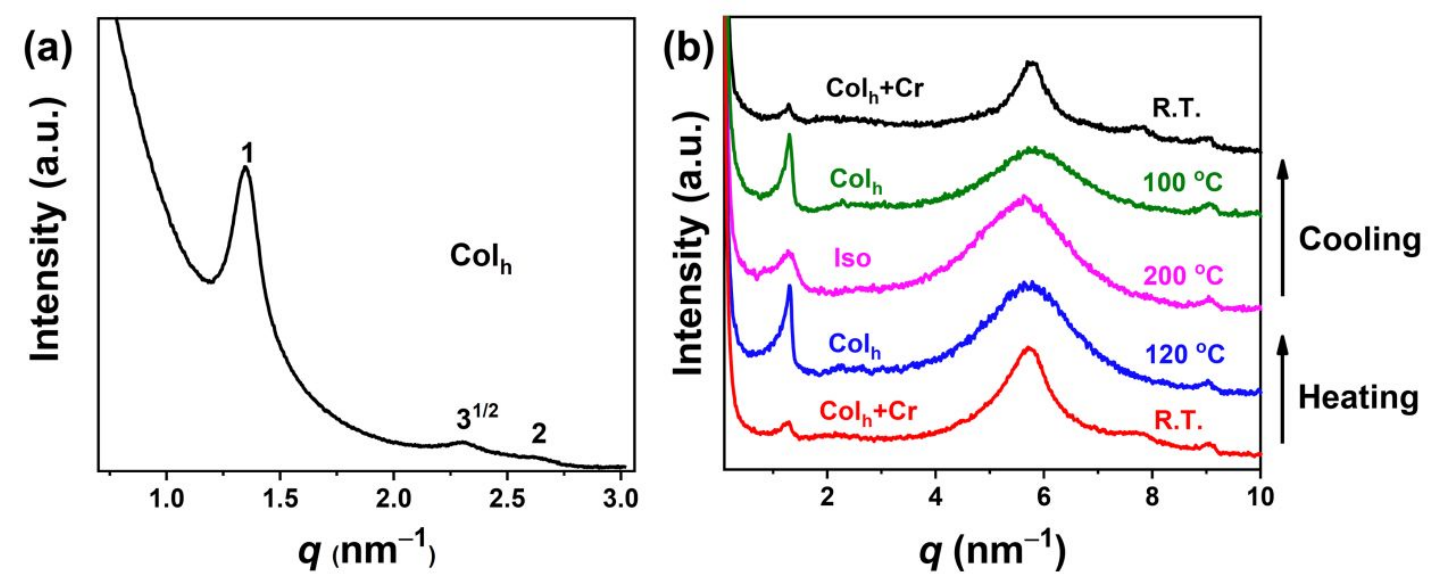

Figure S1. SAXS profiles of PNb12POSS with three diffraction peaks at 1.34, 2.31, and 2.64 $\mathrm{nm}^{-1}$ having a scattering vector ratio of $1: 3^{1 / 2}: 2$, suggesting a $\mathrm{Col}_{\mathrm{h}}$ structure (a) and 1D WAXD profiles of PNb12POSS at different temperatures (b). 


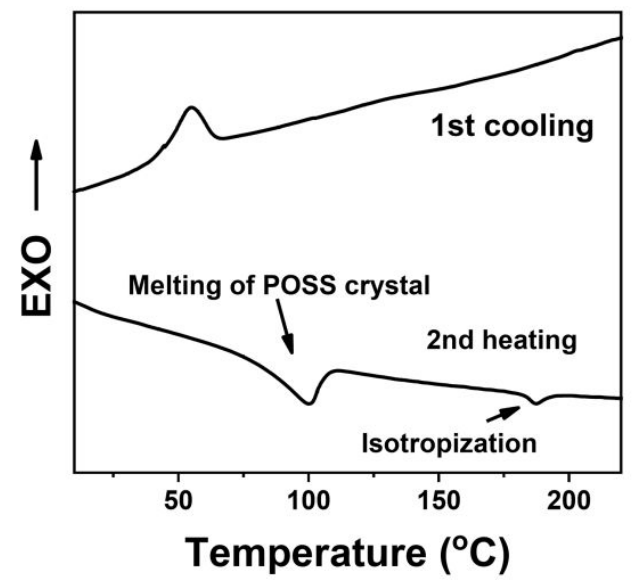

Figure S2. DSC thermograms of PNb12POSS during the first cooling and the subsequent heating processes.



Figure S3. GPC curves of macroinitiators and SmPn samples. 


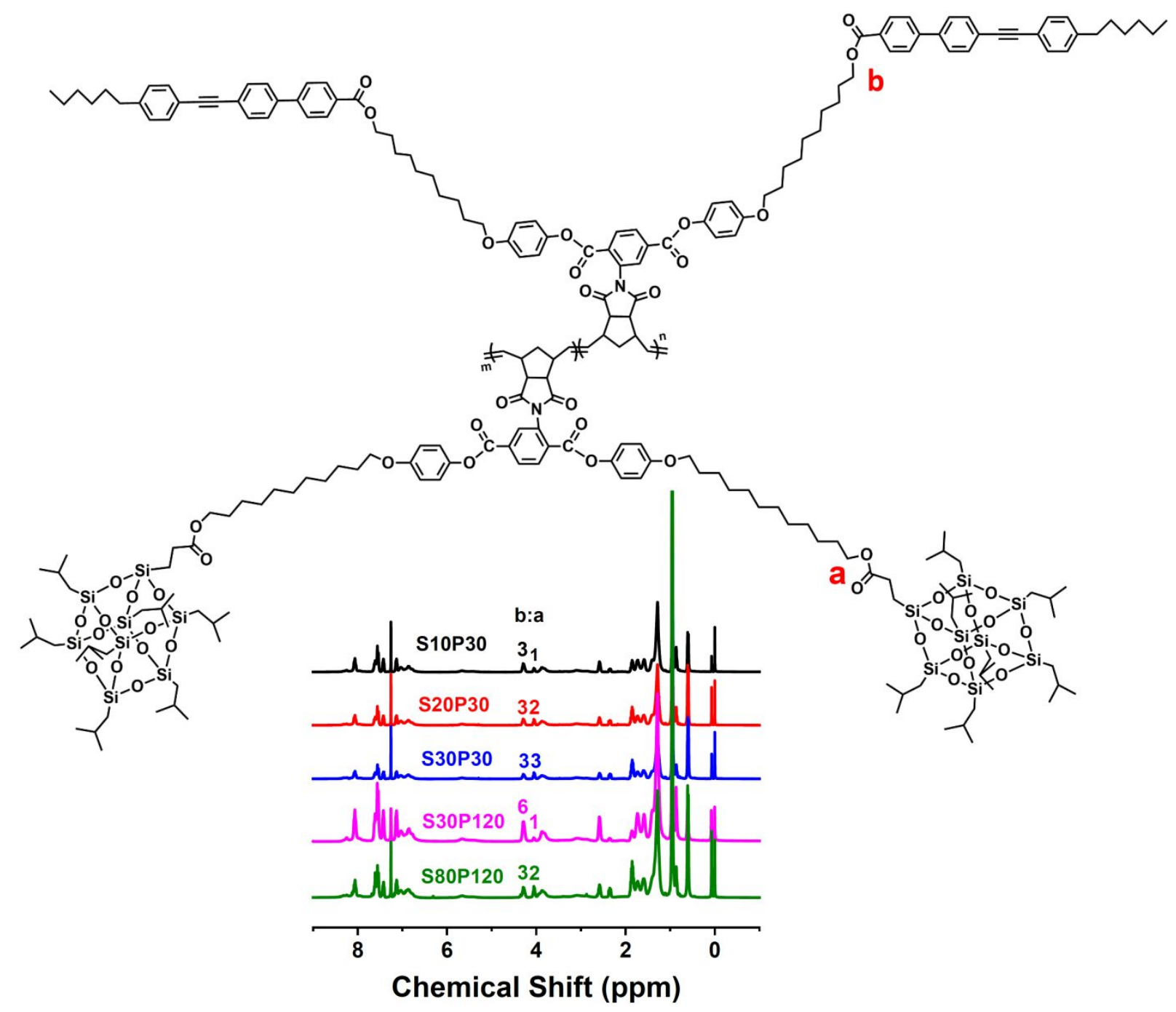

Figure S4. ${ }^{1} \mathrm{H}$ NMR spectra of SmPn samples.

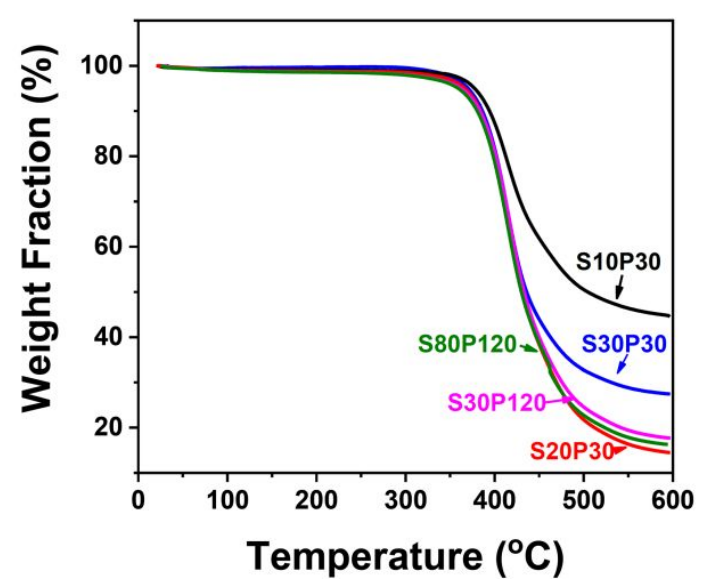

Figure S5. TGA curves of SmPn with a heating rate of $10^{\circ} \mathrm{C} / \mathrm{min}$ under a nitrogen atmosphere. 